SCHWEIZERISCHE ZEITSCHRIFT FÜR HYDROLOGIE BIRKHÄUSER VERLAG BASEL. Vol. XXVI 1964 Fasc. 2

Eidg. Technische Hochschule, Zürich-Fortbildungskurs der EAWAG, 1964

\title{
Die Konsequenzen aus den Ergebnissen der Belebtschlammversuche der EAWAG für die Abwassertechnik
}

Von A. HörLER, EAWAG, Zürich

\section{A. Folgerungen aus den Belebtschlammversuchen für die Wahl der Betriebsparameter und die Dimensionierung der Belüftungsbecken}

\section{Vorbemerkungen}

Die mit grösstem Einsatz der biologischen und chemischen Abteilung der EAWAG und des Personals der Versuchsstation Tüffenwies unter der Versuchsleitung von $\mathrm{K}$. WUHRMANN durchgeführten Versuche bezweckten, den Einfluss von vier Betriebsparametern auf die Reinigungsleistung einer Belebtschlammanlage klarzustellen. Diese Parameter sind I. die hydraulische Raumbelastung, bzw. die Belüftungszeit,

2. die Belebtschlammkonzentration im Belüftungsbecken,

3. der Sauerstoffgehalt im Becken,

4. die Abwassertemperatur (Sommer- und Winterwerte).

Daneben wurde die Überschußschlammproduktion bei den verschiedenen Betriebsarten ermittelt.

Es muss betont werden, dass sämtliche Versuche mit dem relativ dünnen Abwasser der Stadt Zürich durchgeführt wurden mit einem mittleren $\mathrm{BSB}_{5}$-Wert der einzelnen Versuchsabschnitte von 90-I $50 \mathrm{mg} / \mathrm{l}$. Die erhaltenen Beziehungen der einzelnen Parameter zum Reinigungseffekt gelten somit strenggenommen nur für städtische Abwässer ähnlicher Konzentration und Zusammensetzung.

Im folgenden soll überlegt und abgeklärt werden, mit welchen Betriebsparametern eine biologische Reinigungsanlage gefahren werden kann, um einen bestimmten Reinigungseffekt auf wirtschaftlich günstige Weise zu erzielen. Dabei wurde angenommen, dass wie üblich der Reinigungseffekt, gemessen am $\mathrm{BSB}_{5}$, für die Dimensionierung der Anlagen massgebend sei. 
2. Der Einfluss des Sauerstoffgebalts in den Belüftungsbecken auf die Reinigungsleistung einer biologischen Anlage

Die von WUHRManN [r] durchgeführte, statistische Auswertung der mehrjährigen Grossversuche hat ergeben, dass der Sauerstoffgehalt im Beluiftungsbecken für den normalerweise in der Praxis durchgeführten Betrieb von untergeordnetem Einfluss sein muss, wenigstens beim Winterbetrieb, der - wie anschliessend gezeigt wird - in der Regel für die Dimensionierung der Abwasserreinigungsanlagen massgebend ist. Da die Betriebskosten einer Belebtschlammanlage bei Einhaltung höherer Sauerstoffkonzentrationen im Belüftungsbecken rasch zunehmen, wird man aus wirtschaftlichen Gründen bestrebt sein, die Anlagen mit einem nur geringen Sauerstoffgehalt von etwa I-2 $\mathrm{mg} / \mathrm{l} \mathrm{zu}$ betreiben. Wesentlich ist, dass bei hohen Schlammbelastungen im Belüftungsbecken eine leistungsfähige Belüftungseinrichtung installiert wird, die eine starke Turbulenz erzeugt.

\section{Der Einfluss der Abwassertemperatur auf die Reinigungsleistung der biologischen Anlagen}

Die Versuche [I] ergaben für Belüftungszeiten über 2 Stunden, bei Schlammkonzentrationen im Belüftungsbecken über $3000 \mathrm{mg} / \mathrm{l}$ Trockensubstanz, praktisch keinen Unterschied zwischen den Reinigungseffekten der Sommer- und Winterperioden. Bei kleineren Belüftungszeiten und bei niedrigeren Schlammkonzentrationen hingegen wurden im Sommer bei $A b$ wassertemperaturen über $13{ }^{\circ} \mathrm{C}$ bessere Reinigungseffekte erzielt als im Winter bei Abwassertemperaturen unter $\mathrm{II}^{\circ} \mathrm{C}$. Die Unterschiede in den Reinigungseffekten zwischen den Sommer- und Winterwerten nehmen mit zunehmender Schlammbelastung rasch zu (Abb. 2 [r]).

$\mathrm{Da}$ im Winter ungünstigere Reinigungseffekte erzielt werden und da in der Schweiz die Vorfluter zudem im Winter Niederwasser führen, so werden in der Regel die Winterverhältnisse der Dimensionierung von Belebtschlammanlagen zugrunde gelegt werden müssen.

\section{Der Einfluss der Raumbelastung und der Scblammkonzentration im Belüftungs- becken auf die Reinigungsleistung der Anlagen}

a) Begriffe. Die Raumbelastung einer Belebtschlammanlage kann als hydraulische Raumbelastung $R_{h}$ in $\mathrm{m}^{3}$ Abwasser pro $\mathrm{m}^{3}$ Belüftungsraum und Tag ausgedrückt werden oder als sog. biochemische Raumbelastung $R_{B}$ in $\mathrm{kg} \mathrm{BSB}_{5}$ pro $\mathrm{m}^{3}$ Belüftungsraum und Tag. Bezeichnet man mit $s_{R}$ die Konzentration des geklärten Rohwassers, in $\mathrm{kg} \mathrm{BSB}_{5}$ pro $\mathrm{m}^{3}$ Abwasser ausgedrückt, so gilt die Beziehung $R_{B}=s_{R} R_{h}$. 
Die Belüftungszeit $T$ in Stunden errechnet sich aus der hydraulischen Raumbelastung $T=24 / R_{h}$.

Als Schlammbelastung $S_{B}$ bezeichnet man den Quotienten $R_{B}: S$, wobei $S$ die Schlammkonzentration im Belüftungsbecken in $\mathrm{kg}$ Trockensubstanz pro $\mathrm{m}^{3}$ Beckeninhalt bedeutet. Die Schlammbelastung wird gemessen in $\mathrm{kg}$ $\mathrm{BSB}_{5}$ pro kg Schlammtrockensubstanz und Tag.

b) Berecbnung.der Grösse der Belüftungsbecken aus vorgescbriebenen Reinigungseffekten. Die für den projektierenden Ingenieur heute noch wichtigste Beziehung zwischen dem Reinigungseffekt einer Abwasserreinigungsanlage, gemessen am BSB , und der Raumbelastung, bzw. der Schlammbelastung, gibt WUHRMANN [I] in den Abb. I und 2 wieder. Daraus ist ersichtlich, dass sich mit Schlammbelastungen von 0,30 Reinigungseffekte von $90 \%$, gemessen am $\mathrm{BSB}_{5}$, und darüber erzielen lassen, bei Schlammbelastungen von rund $\mathrm{I}, \mathrm{O}$ etwa $85 \%$. Bei höheren Schlammbelastungen ist bereits ein erheblicher Unterschied zwischen den Sommer- und Winterwerten vorhanden. So darf z.B. bei einer Schlammbelastung von 3,0 im Sommer ein Reinigungseffekt von $80 \%$ erwartet werden, im Winter nur noch etwas über $70 \%$. In Abb. I sind die nämlichen Kurven wiedergegeben, ergänzt durch Werte bei kleinsten Schlammbelastungen [4].

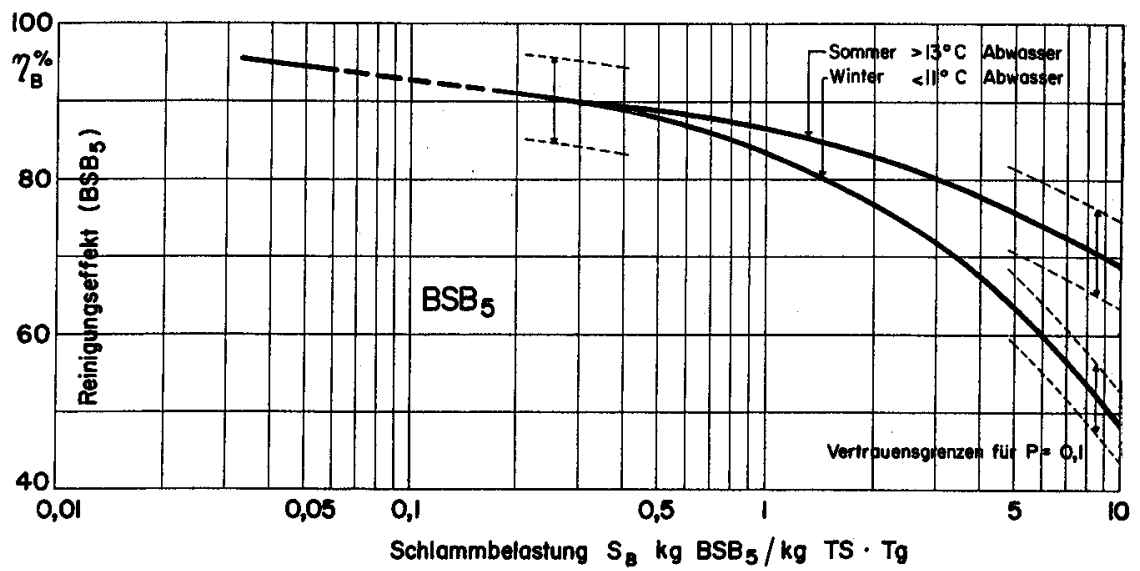

Abbildung 1

Beziehung zwischen Reinigungseffekt $\eta_{B}$ und Schlammbelastung $S_{B}$ nach WuHRMAnN [1], ergänzt bis zu $S_{B}=0,035$ aus den Kombibeckenversuchen [4].

Der Zusammenhang zwischen der Schlammbelastung im Belüftungsbecken und dem Reinigungseffekt einer Abwasserreinigungsanlage, gemessen am $\mathrm{BSB}_{5}$, sei an zwei Beispielen erläutert. Vorweg sei betont, dass es 
sich um Vergleichsberechnungen handelt, wobei die Tagesmittelwerte zur Dimensionierung verwendet wurden. Die Resultate dürfen somit nicht unbesehen zur Bemessung von Anlagen übernommen werden.

Aus der Tab. I geht hervor, dass die Vorschrift eines bestimmten Reinigungseffektes noch keineswegs den Inhalt des Beluiftungsbeckens festlegt. Hingegen ergibt die Wahl von Schlammkonzentrationen im Belüftungsbecken, die nicht unter $\mathrm{I} \mathrm{kg}$ Trockensubstanz pro $\mathrm{m}^{3}$ Becken und praktisch kaum über $5 \mathrm{~kg} / \mathrm{m}^{3}$ liegen werden - konventionelle Betriebsweise vorausgesetzt - eine Begrenzung der Beckengrösse. Um einen 8oprozentigen Reinigungseffekt zu erreichen, genügen somit Becken mit $1 / 2$ bis I Stunde Belüftungszeit. Bei vorgeschriebenen Reinigungseffekten von $90 \%$ müsste bei $1 / 2$ Stunde Belüftungszeit eine Schlammkonzentration von $17,6 \mathrm{~kg} / \mathrm{m}^{3}$ zur Verfügung stehen, was sich praktisch nicht erreichen lässt. Auch eine Schlammkonzentration von $8,8 \mathrm{~kg} / \mathrm{m}^{3}$, wie sie bei einer Stunde Belüftungszeit erforderlich wäre, wird nur in ausserordentlichen Fällen erreicht werden können. Becken mit nur einer Stunde Belüftungszeit oder weniger scheiden deshalb für die Erzielung höherer Reinigungseffekte in der Regel aus.

Tabelle 1

Annahmen: geklärtes Abwasser. Tagesanfall pro Einwohner: Abwassermenge 450 l/E Tag, Biochemischer Sauerstoffbedarf $B S B_{5} 50 \mathrm{~g} / E$ Tag, Abwasserkonzentration (BS $\left.B_{5}\right)$ $=50000: 450=110 \mathrm{mg} / \mathrm{l}$

Fall I: Vorgeschriebener Reinigungseffekt im Tagesmittel $=80 \%$ (Winterwert)

\begin{tabular}{|c|c|c|c|c|c|}
\hline $\begin{array}{l}\text { Belüftungszeit } T \text { (gewählt) } \\
\text { Hydr. Raumbelastung } R_{h}\end{array}$ & $48^{1 / 2}$ & $\begin{array}{r}1 \\
24\end{array}$ & $16^{1 / 2}$ & $\begin{array}{r}2 \\
12\end{array}$ & $\begin{array}{l}\text { Stunden } \\
\mathrm{m}^{3} / \mathrm{m}^{3} \text { Tag }\end{array}$ \\
\hline $\begin{array}{l}\text { Einw. pro } \mathrm{m}^{3} \text { Becken } \\
=R_{h}: 0,450 \mathrm{~m}^{3} / \mathrm{E} \text { Tag } \\
\text { Biochem. Raumbelastung } R_{B}\end{array}$ & 107 & 53 & 36 & 27 & $\mathrm{E} / \mathrm{m}^{3}$ \\
\hline $\begin{array}{l}=R_{h} \cdot 0,110 \mathrm{~kg} / \mathrm{m}^{3} \\
\text { Schlammbelastung für } \eta_{B}\end{array}$ & 5,28 & 2,64 & 1,76 & 1,32 & $\mathrm{~kg} \mathrm{BSB} / \mathrm{m}^{3} \mathrm{Tag}$ \\
\hline $\begin{array}{r}=80 \% \text { (aus Diagramm 1) } \\
\text { Erf. Schlammkonzentration }\end{array}$ & 1,4 & 1,4 & 1,4 & 1,4 & $\mathrm{~kg} \mathrm{BSB}_{5} / \mathrm{kg}$ TS Tag \\
\hline $\begin{array}{l}S=R_{B}: S_{B}, \text { wobei TS }= \\
\text { Trockensubstanz }\end{array}$ & 3,78 & 1,89 & 1,26 & 0,94 & $\mathrm{~kg} \mathrm{TS} / \mathrm{m}^{3}$ \\
\hline
\end{tabular}

Fall II: Vorgeschriebener Reinigungseffekt im Tagesmittel $=90 \%$ (Winterwert)

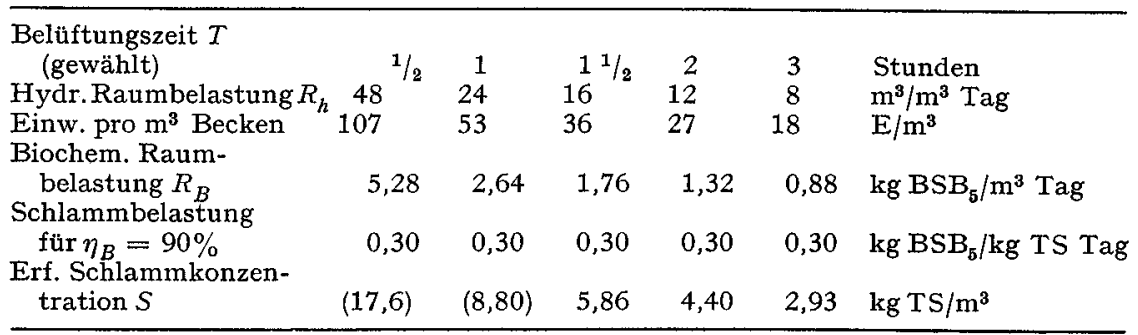


Für eine Stadt von Iooooo Einwohnern würden sich somit bei Verwendung von Tagesmittelwerten unter den gemachten Voraussetzungen folgende Nutzinhalte $\mathcal{F}$ der Belüftungsbecken ergeben:

(Für $T=\mathrm{I}$ Std.: $\mathcal{F}=100000 \mathrm{E} \cdot 0,45 \mathrm{~m}^{3} / \mathrm{E} \mathrm{Tag}: 24=\mathrm{I} 880 \mathrm{~m}^{3}$ ).

Fall I: Reinigungseffekt im Tagesmittel $80 \%$.

7: $940 \mathrm{~m}^{3}$ bei einer Belebtschlammkonzentration von $3780 \mathrm{~g} / \mathrm{m}^{3}$, bzw. F: $\mathrm{I} 880 \mathrm{~m}^{3}$ bei einer Belebtschlammkonzentration von $\mathrm{I} 890 \mathrm{~g} / \mathrm{m}^{3}$. Fall II: Reinigungseffekt im Tagesmittel 90\%.

$\mathcal{F}: 2820 \mathrm{~m}^{3}$ bei einer Belebtschlammkonzentration von $5860 \mathrm{~g} / \mathrm{m}^{3}$, bzw. F: $3760 \mathrm{~m}^{3}$ bei einer Belebtschlammkonzentration von $4400 \mathrm{~g} / \mathrm{m}^{3}$, bzw. F: $5640 \mathrm{~m}^{3}$ bei einer Belebtschlammkonzentration von $2930 \mathrm{~g} / \mathrm{m}^{3}$. Selbstverständlich sind auch alle Zwischenwerte möglich.

Aus Abb. 2 geht die Beziehung zwischen der Belüftungszeit, der Schlammkonzentration und dem Reinigungseffekt, gemessen $\mathrm{am} \mathrm{BSB}_{5}$, für die Winterwerte des Zürcher Abwassers klar hervor. Wir stellen somit fest, dass bei vorgeschriebenem Reinigungseffekt die Wahl der Grösse des Belüftungsbeckens in gewissen, allerdings ziemlich weiten Grenzen noch frei bleibt. Je grösser die Belebtschlammkonzentration bei gegebener Beckengrösse, um so höher ist der erzielbare Reinigungseffekt. Je konzentrierter der Belebtschlamm im Becken ist, um so grösser ist jedoch auch der Sauerstoffverbrauch pro $\mathrm{m}^{3}$ Belüftungsbecken. Im nächsten Abschnitt soll deshalb untersucht werden, ob die Wirtschaftlichkeit des Betriebes die eindeutige Wahl der Belüftungzeit, bzw. der Beckengrösse bestimmt oder wenigstens nahelegt.

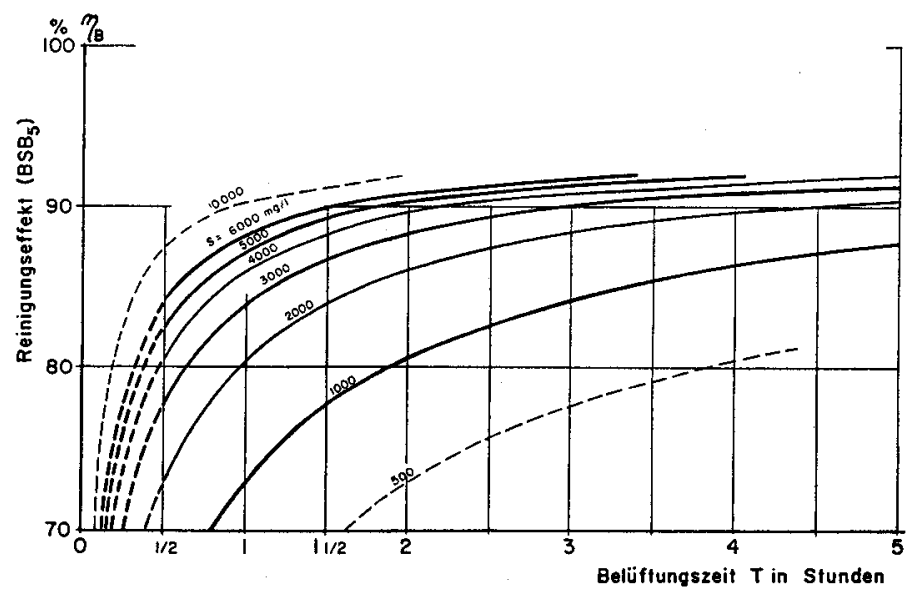

Abbildung 2

Beziehung 7 wischen Belüftungszeit $T$, Schlammkonzentration $S$ und Reinigungseffekt $\eta_{B}$ (Zürcher Abwasser; Winterwerte). 
Um einen weiteren Einblick in die Verhältnisse, namentlich hinsichtlich der Schlussfolgerungen für die Praxis zu gewinnen, wurden die von WUHRMANN [I] beschriebenen Versuchsergebnisse noch nach einem andern Gesichtspunkt aufgetragen. HUNKEN [2] hat in seiner Dissertation darauf hingewiesen, dass die Abwasserreinigung um so rascher vor sich geht, je mehr Organismenmasse pro Raumeinheit im Belüftungsbecken vorhanden ist und je länger diese Masse mit dem Abwasser in Berührung bleibt. Als Masszahl für die Organismenmasse schlagen HUNKEN und KLAUS IMHOFF [3] die Schlammtrockensubstanz vor. Mit andern Worten: die Reinigungswirkung einer Belebtschlammanlage ist vom Produkt $S T$ abhängig, wobei $S$ die Trockensubstanz des Belebtschlamms in $\mathrm{kg} / \mathrm{m}^{3}$ Belüftungsbecken und $T$ die Belüftungszeit in Stunden darstellt. Zwischen dem Ausdruck $S T$ und der Schlammbelastung besteht die Gleichung

$$
S T=24 s_{R} / S_{B} \quad \text { Einheit: } \mathrm{kg} \mathrm{h} / \mathrm{m}^{3} .
$$

Das Produkt $S T$ bedeutet Masse mal die Zeit, in der diese Masse ihre Arbeit verrichtet. Es soll deshalb im folgenden kurz als Scblammarbeit bezeichnet werden.

Die Schlammarbeit ist eine Funktion des Reinigungseffektes und der Abwassertemperatur. Sie dürfte zudem noch von der Abwasserkonzentration abhängig sein. Abb. 3 gilt somit für mittlere Abwasserkonzentrationen von etwa 100-125 mg/ BSB $_{5}$ (Zürcher Abwasser). In Abb. 3 ist der Reini-

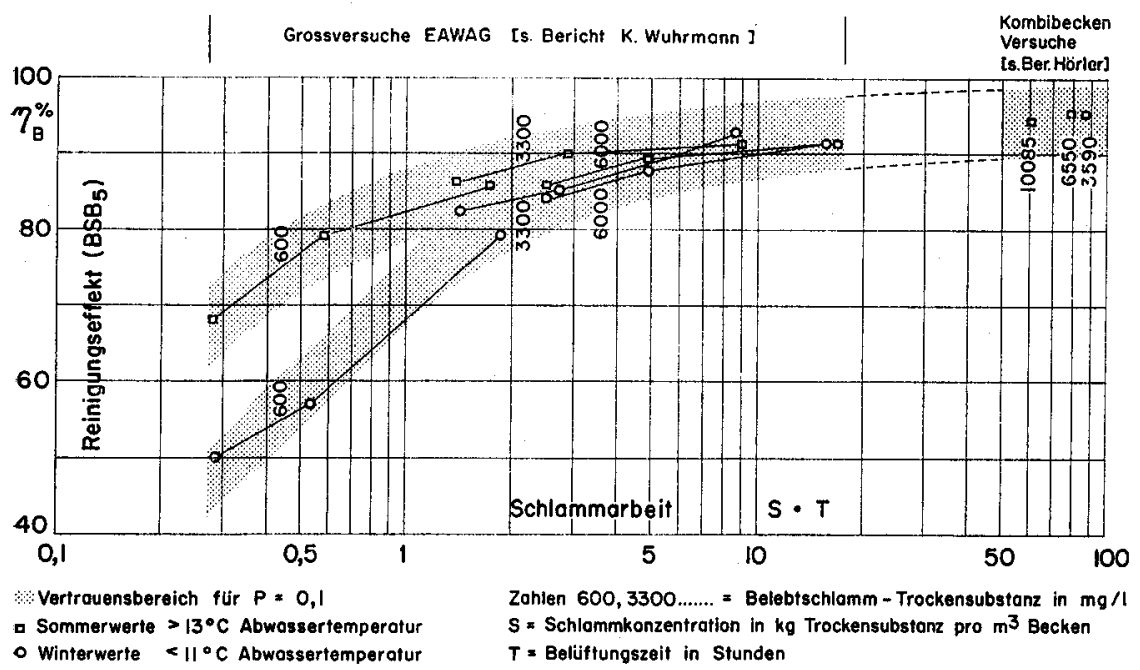

Abbildung 3

Beziehung zwischen Reinigungseffekt $\eta_{B}$ und Schlammarbeit $S T$. 
gungseffekt der Schlammarbeit gegenübergestellt. Infolge der Gleichung ist unter der Voraussetzung konstanter Abwasserkonzentration die Schlammarbeit der reziproken Schlammbelastung proportional. Der Verlauf der Beziehungsbereiche zwischen diesen Variablen ist deshalb den entsprechenden Kurven in Abb. 2 in [I] ähnlich. Der Bereich wurde noch ergänzt durch die ebenfalls mit dem Abwasser der Stadt Zürich durchgef uihrten Kombibeckenversuche [4]. Diese sind bei mittleren Abwassertemperaturen von I2,5-I7,0 ${ }^{\circ} \mathrm{C}$ durchgeführt worden. Die drei Werte stellen Mittel aus je I5 24stündigen Mischproben dar, mit Standardabweichungen von etwas über $\pm 2 \%$.

Aus Abb. 3 ist ersichtlich, dass z. B. im Sommer ein Reinigungseffekt von $86 \%$ erzielt werden kann, sowohl mit einer Schlammkonzentration von $600 \mathrm{mg} / \mathrm{l}$ als auch von $3300 \mathrm{mg} / \mathrm{l}$. Im ersten Fall ist dazu eine Belüftungszeit $T$ von $\mathrm{I}, 5: 0,6 \mathrm{~kg} / \mathrm{m}^{3}=2,5$ Stunden erforderlich, im zweiten Fall genügen $\mathrm{I}, 5: 3,3 \mathrm{~kg} / \mathrm{m}^{3}=0,45$ Stunden oder $27 \mathrm{~min}$. Wir können somit im vorliegenden Fall gleiche Reinigungseffekte erzielen mit Belüftungsbecken, deren Grösse zwischen I und über 5 schwanken kann. Wir stellen fest, dass die Schlammkonzentration im Belüftungsbecken der Belüftungszeit als gleichwertige Grösse zugeordnet ist.

\section{Erforderlicher Sauerstoffeintrag und notwendige Luftmenge für das Belïftungs- becken; Energiebedarf}

Der erforderliche Sauerstoffeintrag $W_{c}$ beträgt nach WUHRMANN [5]

$$
W_{c}=\frac{c_{s}}{c_{s}-c}\left[Q_{o_{e}} S+0, \mathrm{II} \frac{R_{h}}{r_{q}}\left(\mathrm{I}-Y_{S}\right)\left(s_{R_{C}}-s_{C}\right)+0, \mathbf{I} 4 R_{h}\left(s_{R_{N}}-s_{N}\right)\right] \text {, }
$$

wobei $W_{c}$ erforderlicher Sauerstoffeintrag bei einer bestimmten Temperatur in $\mathrm{g} \mathrm{O}_{2} / \mathrm{m}^{3}$ Becken und Std.,

$c_{s} \quad$ Sauerstoffsättigung des Abwassers bei der gewählten Temperatur und

$i \quad$ Sauerstoffgehalt im Belüftungsbecken bei derselben Temperatur, beide Werte in $\mathrm{g} \mathrm{O}_{2} / \mathrm{m}^{3} \mathrm{Abwasser}$,

$Q_{o_{e}} \quad$ Endogene Atmung (Grundatmung) des Belebtschlamms bei der gewählten Temperatur in $\mathrm{g}_{2} / \mathrm{kg}$ TS h,

$S \quad$ Schlammkonzentration in $\mathrm{kg}$ Trockensubstanz $(\mathrm{TS}) / \mathrm{m}^{3}$ Becken,

$R_{h} \quad$ hydraulische Raumbelastung in $\mathrm{m}^{3} \mathrm{Abwasser} / \mathrm{m}^{3}$ Becken Tag,

$r_{q} \quad$ Respirationskoeffizient,

$\stackrel{q}{Y}_{S} \quad$ Ausnützungsfaktor für das Substrat, soweit er zur Überschußschlammbildung führt, in $\mathrm{g}$ Überschußschlamm pro g eliminiertes Substrat.

$s_{R_{C}}$ bzw. $s_{C}$ Konzentration an organischem Kohlenstoff im geklärten Rohwasser, bzw. im gereinigten $A$ bwasser in $\mathrm{g} / \mathrm{m}^{3}$ Abwasser,

$s_{R_{N}}$ bzw. $s_{N}$ Ammoniumkonzentration im geklärten Rohwasser, bzw. im gereinigten Abwasser (Nitrifikation) in $\mathrm{g} / \mathrm{m}^{3}$ Abwasser. 
Die Berechnung des erforderlichen Sauerstoffeintrags und des Luftbedarfs für die beiden Fälle I und II geht aus der Tab. 2 hervor. Unter der Annahme, dass pro m Eintragstiefe der Luft mit etwa $4 \%$ Sauerstoffausnützung gerechnet werden darf, dass somit rund II gSauerstoff pro $\mathrm{m}^{3} \mathrm{Luft}$ in Lösung gehen, erhält man die in der Tab. 3 aufgeführten Luftmengen. Bei grösseren Einblastiefen verringern sich die erforderlichen Luftmengen etwa umgekehrt proportional zur Einblastiefe. Da der Energiebedarf vom Produkt Luftmenge mal Einblastiefe abhängt, so ist dieser in erster Annäherung von der Tiefe des Lufteintrags unabhängig. Als spezifischer Energiebedarf wurde mit $5,5 \mathrm{Wh} / \mathrm{m}^{3}$ eingebrachter Luft und pro m Einblastiefe gerechnet [6]. Die erhaltenen Werte für den Energiebedarf gehen aus der 'Tab. 3 hervor.

Tabelle 2

Erforderlicher Sanerstoffeintrag $=$ Sauerstoffbedarf bei $10^{\circ} \mathrm{C}$ (Winterwerte, Zürcher $A b$ wasser)

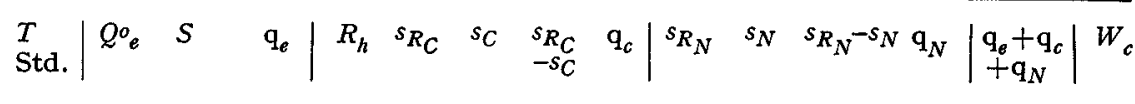

Fall I: Reinigungseffekt $=80 \%$ (siehe Abb. 4)

\begin{tabular}{l|rrr|rrrrr|rrrr|r|r|r}
$1 / 2$ & 5,4 & 3,78 & 20 & 48 & 70 & 26 & 44 & 109 & 10 & 10 & 0 & 0 & 129 & 158 \\
1 & 6,3 & 1,89 & 12 & 24 & 70 & 26 & 44 & 54 & 10 & 10 & 0 & 0 & 66 & 81 \\
$1^{1} / 2$ & 6,7 & 1,26 & 8 & 16 & 70 & 26 & 44 & 36 & 10 & 10 & 0 & 0 & $\mathbf{4 4}$ & 55 \\
2 & 6,8 & 0,94 & 6 & 12 & 70 & 26 & $\mathbf{4 4}$ & 27 & 10 & 10 & 0 & 0 & 33 & $\mathbf{4 1}$
\end{tabular}

Fall II: Reinigungseffekt $=90 \%$ (siehe Abb. 5)

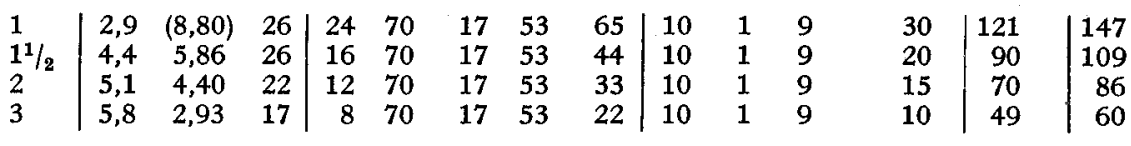
1) 2)
2)
3)
4)
5)
$\left.{ }^{6}\right)$

Bemerkungen:

$W_{c}=\frac{c_{s}}{c_{s}-c}\left(q_{e}+q_{c}+q_{N}\right)$

1) Siehe [5], Abb. 2 (auf $10^{\circ} \mathrm{C}$ umgerechnet).

2) Siehe Tab. 1.

3) Effekt $=63 \%$ (Fall I), bzw. 75\% (Fall II aus Abb. $2[1]$ ).

4) $0,11 \frac{\left(1-Y_{s}\right)}{r_{q}}=\frac{0,11 \cdot(1-0,65)}{0,75}=0,0514$.

5) Abfluss $=10 \mathrm{~g} / \mathrm{m}^{3}$ ohne Nitrifikation, bzw. $1 \mathrm{~g} / \mathrm{m}^{3}$ mit Nitrifikation.

6) $\frac{c_{s}}{c_{s}-c}=\frac{11}{11-2}=1,22$. 


$$
\eta_{8}=80 \%
$$

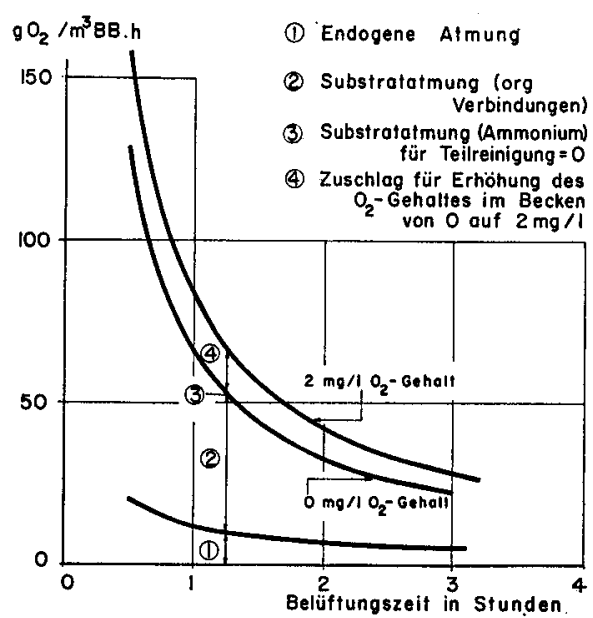

Abbildung 4

Sauerstoffbedarf pro $\mathrm{m}^{3}$ Belüftungsbecken und Stunde bei $2 \mathrm{mg} / \mathrm{O}_{2}$-Gehalt im Mischwasser (Abwasser + Belebtschlamm) bei $10^{\circ} \mathrm{C}$ und $730 \mathrm{~mm} \mathrm{Hg}$. - Reinigungseffekt $=80 \%$.

$$
\eta_{B}=90 \%
$$

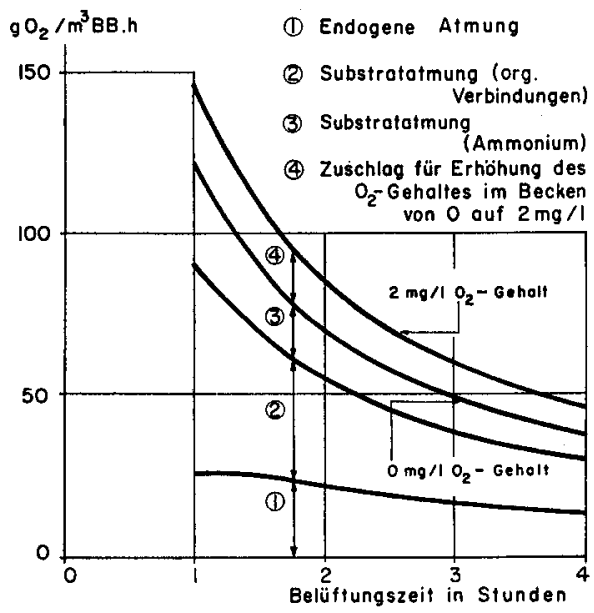

Abbildung 5

Sauerstoffbedarf pro $\mathrm{m}^{3}$ Belüftungsbecken und Stunde bei $2 \mathrm{mg} / 1 \mathrm{O}_{2}$-Gehalt im Mischwasser bei $10^{\circ} \mathrm{C}$ und $730 \mathrm{~mm} \mathrm{Hg}$. - Reinigungseffekt $=90 \%$. 
Aus der Tab. 3 und der zugehörigen Abb. 6 geht hervor, dass bei 8oprozentigem Reinigungseffekt der Energiebedarf bei den üblichen Belüftungszeiten $(T \sim$ I Stunde) praktisch unabhängig von der Belüftungszeit und damit auch von der Beckengrösse ist. Bei Reinigungseffekten von $90 \%$ hingegen ergeben sich mit zunehmender Belüftungszeit nicht nur teurere Becken, sondern auch höhere Energiekosten.

Tabelle 3

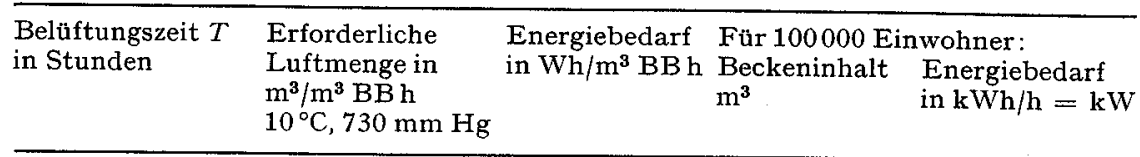

Fall I: Reinigungseffekt $80 \%$

$\begin{array}{lrrrr}1 / 2 & 14,4 & 79,2 & 940 & 74 \\ 1^{1} / 2 & 7,4 & 40,7 & 1880 & 76 \\ 2 & 5,0 & 27,5 & 2820 & 77 \\ & 3,7 & 20,4 & 3760 & 77\end{array}$

Fall II : Reinigungseffekt $90 \%$

\begin{tabular}{lcccc}
1 & 13,4 & 73,8 & 1880 & 139 \\
$\mathbf{1}^{1 / 2}$ & 9,9 & 54,4 & 2820 & 153 \\
2 & 7,7 & 42,4 & 3760 & 159 \\
3 & $\mathbf{5 , 4 5}$ & 30,0 & 5640 & 169 \\
\hline
\end{tabular}

$\mathrm{BB}=$ Belüftungsbecken

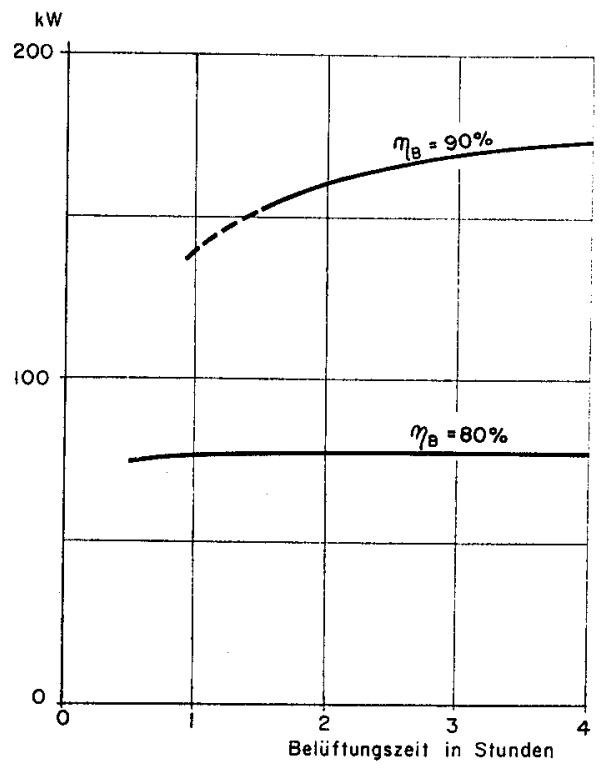

Abbildung 6

Energiebedarf für die Belüftung des Abwassers für eine Stadt von 100000 Einwohnern (Tagesmittelwerte; Abwasser der Stadt Zürich). 


\section{Der Rücklaufscblamm}

Aus Abschnitt 4 geht hervor, dass der Reinigungseffekt einer Belebtschlammanlage gemessen am $\mathrm{BSB}_{5}$ um so grösser ist, je länger die Belüftungszeit und je höher die Belebtschlammkonzentration gewählt wird. Wir haben somit alles Interesse daran, mit hohen Schlammgehalten im Belüftungsbecken zu fahren. Offenbar stehen zwei Mittel zur Verfügung, um diesen Schlammgehalt zu vergrössern: Erhöhung der Rücklaufschlammmenge und Erhöhung der Konzentration des Rücklaufschlamms.

Sofern wir den Schwebestoffgehalt des vorgeklärten Abwassers vernachlässigen, ist der Schlammgehalt im Belüftungsbecken (die Eigenproduktion im Belüftungsbecken während der Belüftungszeit wird im folgenden ausser acht gelassen) nur abhängig vom Rücklaufschlammverhältnis und der im Nachklärbecken erreichbaren Schlammkonzentration. Bezeichnen wir mit $Q_{T}$ den der Dimensionierung zugrunde gelegten Trockenwetteranfall und mit $Q_{R S}$ die Rücklaufschlammenge, so wird mit $Q_{R S}: Q_{T}=n$ bei Anwendung der Mischungsrechnung die Schlammkonzentration $S$ im Belüftungsbecken $S=m \cdot S_{R S} /(\mathrm{I}+m)$, wenn mit $S_{R S}$ die Konzentration des Rücklaufschlamms bezeichnet wird. Wird $Q_{R S}$ konstant gehalten, während der Trockenwetteranfall den normalen Tagesschwankungen unterworfen ist, dann verändert sich $m$, und damit die Schlammkonzentration. Mit diesen Schwankungen wollen wir uns jedoch im folgenden nicht befassen, auch

Tabelle 4

Erforderliche Rücklaufschlammenge in \% des Trockenwetteranfalls zur Aufrechterhaltung einer bestimmten Schlammkonzentration S im Belüttungsbecken

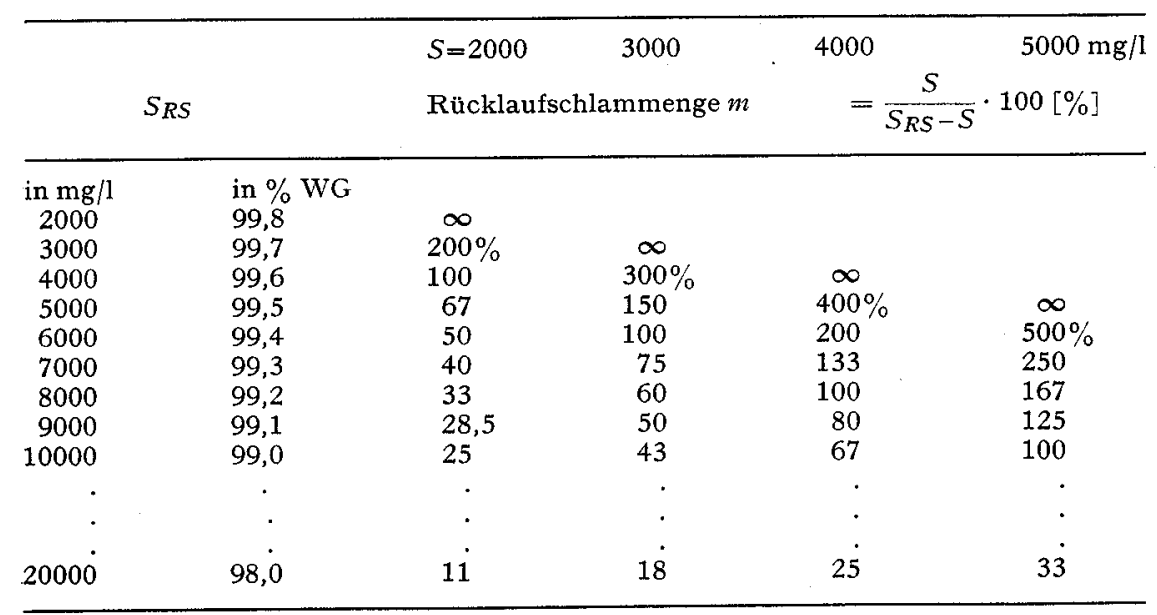

$\mathrm{WG}=$ Wassergehalt 
nicht mit den Verhältnissen bei Regenwetter, sondern annehmen, dass der rechnerische Trockenwetteranfall vorhanden sei. Nach der Mischungsrechnung ergeben sich dann folgende, in der Tab. 4 aufgeführte Rücklaufschlammengen in \% des Trockenwetteranfalls bei gewünschten Schlammgehalten $S$ im Belüftungsbecken und vorhandenen Rücklaufschlammkonzentrationen $S_{R S}$.

Aus Tab. 4 ist ersichtlich, dass eine wirkungsvolle Eindickung des Rücklaufschlamms im Nachklärbecken unbedingt erforderlich ist, sobald mit höheren Schlammkonzentrationen $S$ im Belüftungsbecken gefahren werden muss und abnormal grosse Rücklaufschlammpumpwerke vermieden werden sollen. Sonst dürften die Energie- und Betriebskosten dieser Pumpwerke geringer Förderhöhen nicht mehr vernachlässigt werden.

\section{7. Überscbußscblammproduktion}

Nach A.L. DownING und A.P.HoPwoon [7] ist die Überschußschlammproduktion von der Belüftungszeit abhängig: je länger die Belüftungszeit, um so geringer der Anfall an Überschußschlamm. Als Beziehung zwischen der Überschußschlammproduktion und der Belüftungszeit wird, unab hängig von der Schlammkonzentration im Belüftungsbecken, angegeben:

$$
\frac{\text { Produzierte Belebtschlammasse }}{\text { Zugefuihrte } \mathrm{BSB}_{5} \text {-Menge }}=0, \mathrm{I}+\frac{0,9}{\sqrt{T}},
$$

wobei $T=$ Belüftungszeit in Stunden (Bereich $\mathrm{I}<T<7$ ).

Da der Überschußschlamm die Kosten der Schlammbehandlung beeinflusst und somit die Wirtschaftlichkeit der Anlage, wurde von Dr. WuHRMANN ebenfalls die Beziehung zwischen Belüftungszeit und Überschußschlammenge mit der Varianzanalyse statistisch untersucht. Dabei zeigte sich, dass bei den für die Versuche gewählten Belüftungszeiten (25 min, so min, 2,4 Stunden) keine gesicherte Beziehung zwischen der Belüftungszeit, der Schlammkonzentration und der Überschußschlammenge gefunden werden konnte. Die Úberschußschlammproduktion wird deshalb für den Untersuchungsbereich als konstant angenommen.

\section{B. Wahl der Höheṇlage und der Länge der Ủberfallkanten bei Belüftungsbecken}

\section{Vorbemerkungen}

Bei der Durchsicht von Projekten von Belebtschlammanlagen stellt man immer wieder fest, dass eine gewisse Unsicherheit besteht bezüglich der Wahl der Überfallkantenlängen und auch deren Kronenhöhen. Bei den im 
Betrieb befindlichen Anlagen hat man ebenfalls das Empfinden, dass der Klärmeister die Erhöhung oder Erniedrigung der Überfallkanten willkürlich vornimmt mangels Instruktionen. Wir stellen uns deshalb die Frage, ob Höhenlage und Überfallkantenlänge wirklich frei gewählt werden können oder ob diese bestimmten Bedingungen unterworfen sind. Dabei sollen Becken mit gesteuerten Überfällen in Abhängigkeit von der Sauerstoffkonzentration im Belüftungsbecken aus unsern Betrachtungen ausgeschieden werden.

\section{Die Scbwankungen des Tagesanfalls}

Der Abwasseranfall auf einer Abwasserreinigungsanlage ist ständigen Schwankungen unterworfen. Die Abwassermengen können an einzelnen Tagesstunden das I, 5 fache des Tagesmittels übersteigen, um während der Nacht auf die Hälfte des mittleren Tagesanfalls herunterzusinken. Die Konzentration des Abwassers, gemessen in $\mathrm{mg} / \mathrm{l} \mathrm{BSB}_{5}$, ist während der einzelnen Tagesstunden ähnlichen Schwankungen unterworfen. Die Differenzen in den Stoffmengen, also des Produktes Wassermenge mal Konzentration, sind deshalb noch erheblich grösser. Diese Verhältnisse wurden für drei Untersuchungstage (Stadt Winterthur) in Abb. 7 graphisch dargestellt.

\section{Abbildung 7}

Schwankungen der Abwassermenge $Q$ in $\mathrm{m}^{3} / \mathrm{h}, \mathrm{bzw}$. des $\mathrm{BSB}_{6}$ in $\mathrm{kg} \mathrm{O}_{2} / \mathrm{h}$ während $n$ ununterbrochenen Stunden. Abwasser der Stadt Winterthur.

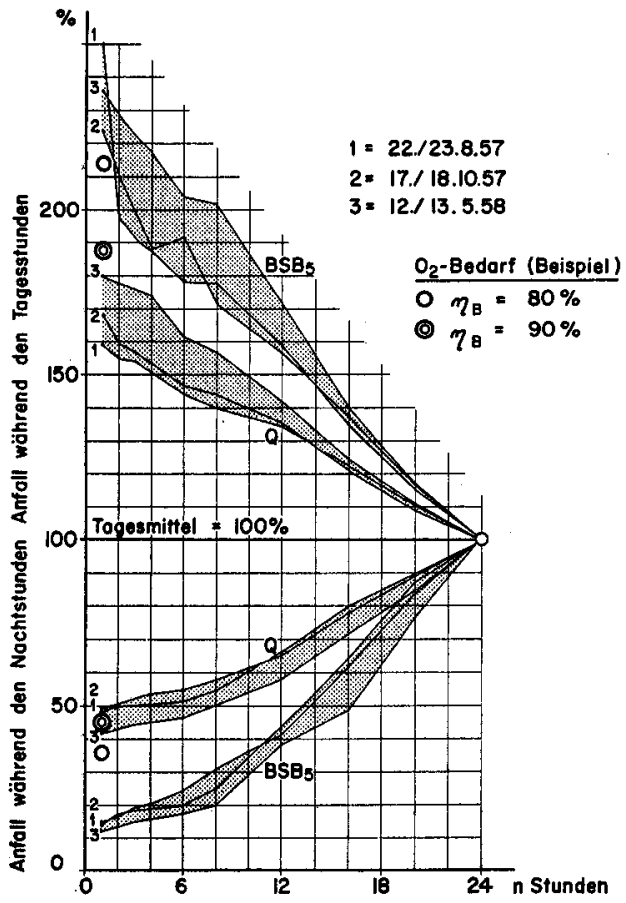


Die Mittelwerte der Abwassermenge und des $\mathrm{BSB}_{5}$ über die 24 Tagesstunden wurden $=100 \%$ gesetzt. Aus Abb. 7 gehen die grössten Abweichungen während $\mathrm{I}, 2, \ldots n$ ununterbrochenen Stunden zur Tages- und Nachtzeit gegenüber dem Tagesmittel hervor. Es stellt sich nun die Frage, wie sich die einzelnen Belüftungssysteme bezüglich des Sauerstoffeintrags zum jeweiligen Sauerstoff bedarf während des Tagesverlaufs verhalten.

\section{Becken mit Druckluftbelïftung}

Da die Gebläse, die die Druckluft liefern, im stabilen Bereich ihrer Kennlinien mit steigendem Druck die Luftmenge reduzieren, wird die gelieferte Luftmenge bei starrer Überfallkante während der Tagesstunden sinken und während der Nachtstunden ansteigen. Die Sauerstoffversorgung erfolgt somit gegenläufig zum Bedarf. Eine Regelung durch Hinzuschalten von Gebläseeinheiten während der Tagesstunden drängt sich auf. Wird dies unterlassen, dann wird man bestrebt sein müssen, den Einfluss dieser gegenläufigen Schwankungen möglichst klein zu halten. Dies kann bei starren Überfallkanten nur dadurch, und zwar nur in beschränktem Masse, erreicht werden, indem die Überfallkante der Belüftungsbecken möglichst lang gewählt wird, so lange, als dies betriebstechnisch verantwortbar ist.

\section{Becken mit Bürsten- und Stabsalzenbelüftung}

Bei Becken mit Oberfächenbelüftung in Form von Bürsten- oder Stabwalzen liegen die Verhältnisse wesentlich anders. Mit der erhöhten Wassermenge um die Mittagszeit steigt die Úberfallhöhe beim Beckenauslauf an und damit auch die Eintauchtiefe der Walze. Dadurch wird der Sauerstoffeintrag vermehrt, was erwünscht ist. Da durch geeignete Wahl der Höhenlage der Überfallkante und der Überfallänge die Eintauchtiefe der Bürste oder Walze variiert werden kann, so stellt sich die Aufgabe, diese beiden Grössen so zu wählen, dass der Sauerstoffbedarf während der Tages- und Nachtstunden mit dem Sauerstoffeintrag der Walze übereinstimmt. Die Lösung dieser Aufgabe erfolgt schrittweise:

r. Dimensionierung der Belüftungsbecken nach den im Abschnitt A dargelegten Gesichtspunkten.

2. Ermittlung der täglichen Schwankungen des Abwasseranfalls und der Konzentra$\operatorname{tion}\left(Q_{\text {max }}, Q_{\text {min }}, Q_{\text {mittel, }}, \mathrm{BSB}_{5 \text { max, min, miltel }}\right)$.

3. Errechnung des $\mathrm{O}_{2}$-Bedarfs für die zwei Tagesextremwerte und für das Tagesmittel.

4. Wahl der Belüftungseinrichtung und damit auch der Querschnittsfläche des Belüftungsbeckens. 
5. Aus der Beziehung zwischen der Eintauchtiefe und dem $\mathrm{O}_{2}$-Eintrag der Bürste oder Walze bei konstanter Tourenzahl ergibt sich die erforderliche Eintauchtiefe für die drei Betriebszustände zur Deckung des $\mathrm{O}_{2}$-Bedarfs. Dadurch sind die Eintauchtiefen der Walze $b_{\text {max }}, b_{\text {min }}$ und $b_{\text {mittel }}$ festgelegt. Die Überfallhöhen beim Beckenauslauf ergeben sich zu $y+b_{\max }, y+b_{\text {min }}$ und $y+b_{\text {mittel }}$, wobei die Differenz $y$ zwischen Überfallkrone und Unterkante Walze vorerst noch unbekannt ist.

6. Diese Überfallhöhen lassen sich durch geeignete Wahl der Überfallänge $x$ steuern. Die zwei Unbekannten $y$ und $x$ können durch zwei Gleichungen ermittelt werden. Dazu dienen z.B. die Überfallformeln für die beiden extremen Werte $Q_{\max }$ und $Q_{\min }$. 7. Es ist zu kontrollieren, ob bei den errechneten Werten $y$ und $x$ für $Q_{\text {mittel }}$ der durch die Walze gelieferte Sauerstoffeintrag genügt oder ob eventuell eine Korrektur vorgenommen werden muss.

Der Rechnungsgang soll an einem Beispiel unter der Annahme der Vollbelastung der Anlage erläutert werden. Gewählt wird Fall II mit einem erforderlichen Reinigungseffekt von $90 \%$, gemessen am $\mathrm{BSB}_{5}$ (s. Abschnitt A 4). Es sollen für dieses Beispiel der Ưbersichtlichkeit wegen wieder die Tagesmittelwerte der Dimensionierung zugrunde gelegt werden, gemäss den Gepflogenheiten in den USA. Die Bezeichnungen gehen aus dem Abschnitt A 4 hervor (Winterwerte).

I. Annahmen:

$$
\begin{array}{ll}
\text { Belüftungszeit } & =2 \text { Stunden } \\
R_{h} & =12 \mathrm{~m}^{3} / \mathrm{m}^{3} \text { Tag } \\
\mathrm{BSB}_{5} & =50 \mathrm{~g} / \mathrm{E} \mathrm{Tag} \\
Q_{E} & =450 \mathrm{l} / \mathrm{E} \mathrm{Tag} \\
\mathrm{BSB}_{5} & =\mathrm{IIO} \mathrm{mg} / \mathrm{l} \\
\text { Einwohner pro } \mathrm{m}^{3} \text { Becken } & =12 \mathrm{~m}^{3}: 0,45=26,7 \\
R_{B} & =12 \mathrm{~m}^{3} \cdot \mathrm{IIO} / \mathrm{m}^{3}=1,32 \mathrm{~kg} / \mathrm{m}^{3} \mathrm{Tag} \\
S(\text { Tab. } \mathrm{I}) & =4,4 \mathrm{~kg} / \mathrm{m}^{3}
\end{array}
$$

2. Abwassermenge:

$$
\begin{aligned}
& Q_{\text {mittel }}=12 \mathrm{~m}^{3} / \mathrm{m}^{3} \mathrm{Tag}=0,50 \mathrm{~m}^{3} / \mathrm{m}^{3} \mathrm{~h} \\
& Q_{\max }=170 \% \cdot 0,5=0,85 \mathrm{~m}^{3} / \mathrm{m}^{3} \mathrm{~h} \\
& Q_{\text {min }}=50 \% \cdot 0,5=0,25 \mathrm{~m}^{3} / \mathrm{m}^{3} \mathrm{~h} \\
& \mathrm{BSB}_{5}: \mathrm{BSB}_{\text {mittel }}=\mathrm{I}, 32 \mathrm{~kg} / \mathrm{m}^{3} \mathrm{Tag}=55 \mathrm{~g} / \mathrm{m}^{3} \mathrm{~h} \\
& \mathrm{BSB}_{\max }=230 \% \cdot 55=126 \mathrm{~g} / \mathrm{m}^{3} \mathrm{~h} \\
& \mathrm{BSB}_{\min }=20 \% \cdot 55=\mathrm{II} \mathrm{g} / \mathrm{m}^{3} \mathrm{~h}
\end{aligned}
$$

3. Erforderlicher Sauerstoffeintrag (nach Tab. 2):

Endogene Atmung

Substratatmung:

- Organische Verbindungen

- Ammonium

Total

$\times$ Faktor $1,22(2 \mathrm{mg} / \mathrm{l} \mathrm{O})$

Verhältniszahlen
Max. Mittel Min.

$22 \quad 22 \quad 22 \mathrm{~g} \mathrm{O}_{2} / \mathrm{m}^{3} \mathrm{~h}$

\begin{tabular}{ccc}
76 & 33 & $6,6 \mathrm{~g} \mathrm{O}_{2} / \mathrm{m}^{3} \mathrm{~h}$ \\
35 & $\mathrm{I} 5$ & $3,0 \mathrm{~g} \mathrm{O}_{2} / \mathrm{m}^{3} \mathrm{~h}$ \\
$\mathrm{I} 33$ & 70 & $32 \mathrm{~g} \mathrm{O}_{2} / \mathrm{m}^{3} \mathrm{~h}$ \\
$\mathrm{I} 62$ & 86 & $39 \mathrm{~g} \mathrm{O}_{2} / \mathrm{m}^{3} \mathrm{~h}$ \\
\hline $\mathrm{I} 88 \%:$ & $100 \%:$ & $45 \%$
\end{tabular}


4. Wahl der Walze:

Aluminiumguss, $\varnothing 42 \mathrm{~cm}$ gemäss Versuchsbecken (Versuchsstation Tüffen wies der EAWAG).

Zulässige Belastung pro $\mathrm{m}^{1}$ Walze (brutto) $=$ rd. $\mathrm{I}_{2} \mathrm{~kg} \mathrm{BSB}_{5} /$ Tag.

Beckenquerschnitt $=\frac{12 \mathrm{~kg} / \mathrm{m}^{1} \mathrm{Tag}}{1,32 \mathrm{~kg} / \mathrm{m}^{3} \mathrm{Tag}}=9, \mathrm{I} \mathrm{m}^{3} / \mathrm{m}^{1}=9, \mathrm{r} \mathrm{m} \mathrm{m}^{2}$.

Belastung $\quad=\frac{12 \mathrm{~kg} / \mathrm{m}^{1} \mathrm{Tag}}{0,050 \mathrm{~kg} / \mathrm{E} \mathrm{Tag}}=240$ Einwohner $/ \mathrm{m}^{1}$ Walze.

Tourenzahl gewählt entsprechend erforderlichem Sauerstoffeintrag $=120 \mathrm{U} / \mathrm{min}$. Beziehung zwischen Eintauchtiefe und Sauerstoffeintrag aus Belüftungsversuchen bekannt.

Werte 2. auf $\mathrm{I} \mathrm{m}$ Beckenlänge (pro $\mathrm{m}^{1}$ Walze) umrechnen:

Abwassermenge $\left(9, \mathrm{I} \mathrm{m}^{3} / \mathrm{m}^{1}\right.$ Walze):

$Q_{\text {mittel }}=0,50 \mathrm{~m}^{3} / \mathrm{m}^{3} \mathrm{~h} \cdot 9, \mathrm{I} \mathrm{m^{3 }} / \mathrm{m}^{1}: 3600 \mathrm{~s}=1,261 / \mathrm{s} \mathrm{m}^{1}$

$Q_{\text {max }}=170 \% Q_{\text {mittel }} \quad=2,14 \mathrm{l} / \mathrm{s} \mathrm{m}^{1}$

$Q_{\min }=50 \% Q_{\text {mittel }} \quad=0,63 \mathrm{l} / \mathrm{s} \mathrm{m}^{1}$

5. $\mathrm{O}_{2}$-Bedarf pro $\mathrm{m}$ Walze: (siehe 3.)

Max.: $\quad 162 \mathrm{~g} \mathrm{O}_{2} / \mathrm{m}^{3} \mathrm{~h} \cdot 9, \mathrm{I} \mathrm{m}^{3} / \mathrm{m}^{1}=1470 \mathrm{~g} \mathrm{O}_{2} / \mathrm{m}^{1} \mathrm{~h}$

Mittel: $\quad 86 \mathrm{~g} \mathrm{O}_{2} / \mathrm{m}^{3} \mathrm{~h} \cdot 9, \mathrm{I} \mathrm{m}^{3} / \mathrm{m}^{1}=780 \mathrm{~g} \mathrm{O}_{2} / \mathrm{m}^{1} \mathrm{~h}$

Min.: $\quad 39 \mathrm{~g} \mathrm{O}_{2} / \mathrm{m}^{3} \mathrm{~h} \cdot 9, \mathrm{I} \mathrm{m}^{3} / \mathrm{m}^{1}=355 \mathrm{~g} \mathrm{O}_{2} / \mathrm{m}^{1} \mathrm{~h}$

Der entsprechende Sauerstoffeintrag wird von der Bürstenwalze bei $\mathrm{I} 20$ Touren $/ \mathrm{min}$ geliefert bei folgenden Eintauchtiefen $b$ :

$b_{\text {max }}=\mathrm{I} 3 \mathrm{~cm} ; b_{\text {mittel }}=8 \mathrm{~cm} ; b_{\min }=4 \mathrm{~cm}$.

6. Pro $\mathrm{m}^{1}$ Beckenlänge bzw. Walzenlänge ergeben sich folgende Überfallmengen $Q_{\ddot{u}}$ $\left(Q_{\max }\right.$ und $\left.Q_{\text {min }}\right)$ :

$Q_{\max }=x \cdot 1,85\left(y+b_{\max }\right)^{3 / 2}$

$Q_{\min }=x \cdot 1.85\left(y+b_{\text {min }}\right)^{3 / 2}$;

$0,002 \mathrm{I} 4 \mathrm{~m}^{3} / \mathrm{s}=x \cdot \mathrm{I}, 8 \mathrm{~s}(y+0, \mathrm{I} 3)^{3 / 2}$

Daraus errechnen sich die Werte $y$ und $x$, wie in Bild $8 a$ und $8 b$ dargestellt zu

Kronenhöhe der Überfallkante unterhalb Unterkante Walze $y=3,2 \mathrm{~cm}$.

Länge der Überfallkante pro $\mathrm{m}^{\mathbf{1}}$ Beckenlänge $=\mathrm{I}, 8 \mathrm{~cm}$,

bzw. Länge der Überfallkante pro rooo angeschlossene Einwohner

$=\mathrm{r}, 8 \mathrm{~cm} \cdot 1000 \mathrm{E} / 240 \mathrm{E}=7,5 \mathrm{~cm}$.

7. Kontrolle der Verhältnisse beim mittleren Tagesanfall:

Vorhandene Ein tauchtiefe der Walze:

$$
\begin{array}{rlrl}
Q_{\text {mittel }}=0,00126 \mathrm{~m}^{3} / \mathrm{s}=0,018 \mathrm{~m} \cdot \mathrm{I}, 85 & \left(y+b_{\text {mittel }}\right)^{3 / 2} \\
\left(y+b_{\text {mittel }}\right)^{3 / 2}=0,0378 ; & y+b & =0, \mathrm{II} 3 \mathrm{~m}=\mathrm{II}, 3 \mathrm{~cm} \\
-y & = & -\frac{3,2 \mathrm{~cm}}{8, \mathrm{Im}} \\
b & = &
\end{array}
$$

Bei der gewählten Kronenhöhe der Überfallkante und der Überfallänge beträgt die Eintauchtiefe der Walze im Tagesmittel $=8, \mathrm{I} \mathrm{cm}$. Erforderlich wäre nach 5 eine Eintauchtiefe von $8 \mathrm{~cm}$. 


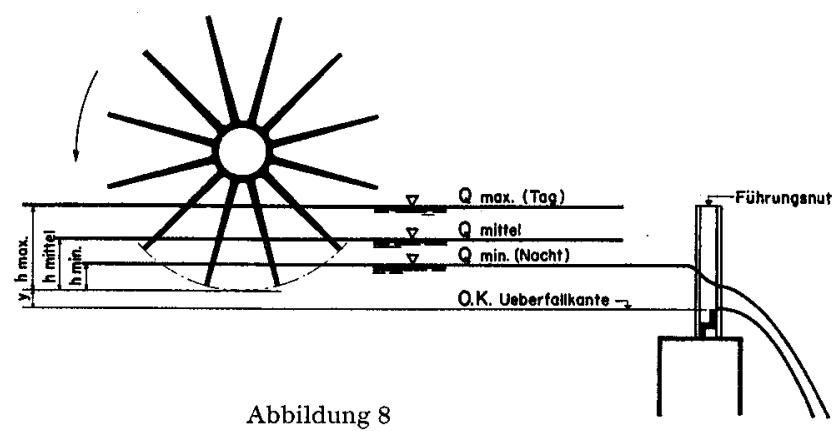

$a$ Schnitt durch Belüftungsbecken mit Bürstenwalze $\varnothing 42 \mathrm{~cm}$ bei Vollbelastung der Anlage (Reinigungseffekt $=90 \%$ )

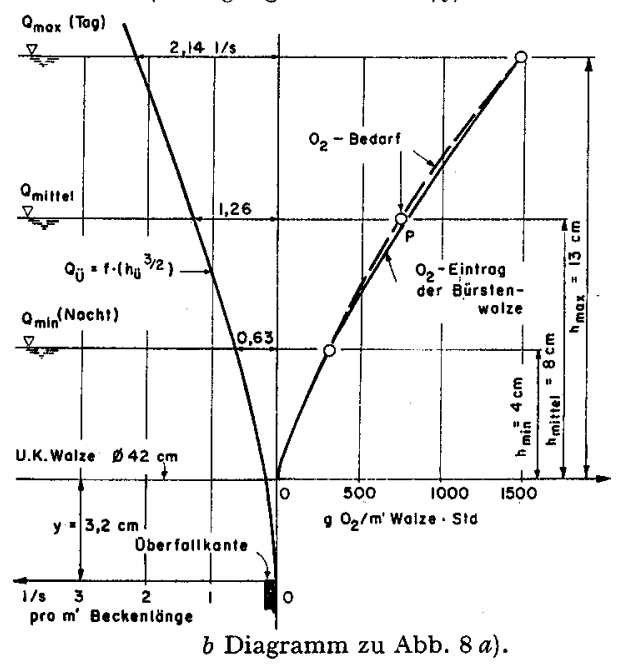

Die gleiche Berechnung wurde ebenfalls für den Fall $\mathrm{I}$, Reinigungseffekt $=80 \%$, durchgeführt. Dabei ergab sich ein Wert $y$ als Höhendifferenz zwischen Überfallkante und Unterkante Walze von $\mathrm{r}, 8 \mathrm{~cm}$ und eine erforderliche Überfallkantenlänge von $3,6 \mathrm{~cm}$ pro $\mathrm{m}^{1}$ Beckenlänge, bzw. von $15 \mathrm{~cm}$ pro tooo angeschlossene Einwohner. Der Wert $y$ hat sich gegenuiber den Verhältnissen bei $90 \%$ Reinigungseffekt beinahe um die Hälfte reduziert, die Ưberfallänge verdoppelt.

\section{G. Zusammenfassung und Schlussfolgerungen für die Praxis}

Die auf Versuchen der EAWAG fussende Veröffentlichung von WUHRMANN [r] und die darauf aufbauenden Berechnungen lassen folgende Schlussfolgerung zu: 
I. Die Untersuchungsresultate wurden erzielt mit dem relativ dünnen Zürcher Abwasser $\left(\mathrm{BSB}_{5}=90-\mathrm{I} 5 \mathrm{mg} / \mathrm{l}\right)$. Die Schlussfolgerungen sind deshalb gültig für Anlagen mit städtischem Abwasser ähnlicher Konzentration und Zusammensetzung.

2. Der Sauerstoffgehalt im Belüftungsbecken ist von untergeordnetem Einfluss auf den Reinigungseffekt einer Belebtschlammanlage, gemessen am $\mathrm{BSB}_{5}$. Wesentlich ist jedoch eine hohe Turbulenz im Belüftungsbecken, namentlich bei hohen Schlammbelastungen.

Eine Beziehung zwischen dem Reinigungseffekt, gemessen $\mathrm{am} \mathrm{BSB}_{5}$, und der Schlammbelastung ist feststellbar. Je grösser die Schlammbelastung, um so kleiner ist der Reinigungseffekt. Für Schlammbelastungen über 0,30 $\mathrm{kg} \mathrm{BSB} 5$ pro $\mathrm{kg}$ Trockensubstanz und Tag sinkt mit zunehmender Belastung der Reinigungseffekt in der kalten Jahreszeit rascher ab als im Sommer. Daraus geht hervor, dass namentlich für Ortschaften mit niedrigen Abwassertemperaturen nur Anlagen mit kleinen Schlammbelastungen projektiert werden sollten. Dieser Schluss dürfte namentlich für die Winterkurorte von Bedeutung sein. Dabei ist zu beachten, dass die angegebenen Winterwerte für eine mittlere Abwassertemperatur von $10^{\circ} \mathrm{C}$ gelten, die Sommerwerte für $16,3^{\circ} \mathrm{C}$ im Mittel. Versuchsresultate bei niedrigeren $\mathrm{Ab}$ wassertemperaturen sind noch zu erheben.

4. Da sich in der kalten Jahreszeit schlechtere Reinigungseffekte ergaben als im Sommer und in der Schweiz die Vorfluter in der Regel im Winter die geringste Wasserführung aufweisen, so sind bei der Beurteilung der Belastbarkeit eines Vorfluters und bei der Festlegung des erforderlichen Reinigungseffektes, bzw. der Schlammbelastung von Abwasserreinigungsanlagen, die Winterverhäl tnisse zu berücksichtigen.

5. Für Abwässer gleicher Konzentration ist der reziproke Wert der Schlammbelastung $S_{B}$ proportional zur Schlammarbeit $S T$. Der Reinigungseffekt eines Belüftungsbeckens hängt somit nicht nur von der Belüftungszeit $T$ ab, sondern in gleichem Masse von der Schlammkonzentration $S$, mit der die Anlage gefahren wird. Wir können deshalb bessere Reinigungsleistungen erzielen entweder durch längere Belüftungszeiten oder ebensogut mit konzentrierteren Belebtschlämmen. Diese grösseren Schlammkonzentrationen können durch grössere Rücklaufschlammengen oder durch Rückpumpen konzentrierterer Schlämme erreicht werden. Da nur die Schlammsubstanz für die Reinigungswirkung wesentlich ist, wird man bestrebt sein müssen, nicht grosse Wassermengen zurückzupumpen, sondern möglichst viel Schlammsubstanz. Das Rückpumpen grosser Wassermengen kann nur dort u. U. erwünscht sein, wo das Abwasser praktisch sauerstofffrei anfällt. 
Der Grad der möglichen Schlammkonzentration ist bedingt durch die Eigenschaften des Schlamms (Mohlman-Index oder besser die Sackungsgeschwindigkeit) und durch die Konstruktion und Betriebsweise des Nachklärbeckens. Es sollte deshalb zukünftig in Praxis und Forschung den Nachklärbecken vermehrte Aufmerksamkeit geschenkt werden.

6. Die bisherigen Berechnungen des Sauerstoff bedarfs bei Teilreinigung, bzw. bei Vollreinigung, waren unbefriedigend. Sie ergaben Unstimmigkeiten gegenüber den tatsächlichen Verhältnissen. Die neue Formel (WuHRMANN [s]) berücksichtigt die auftretenden Reaktionen und entspricht deshalb den Bedürfnissen. Zu ihrer Handhabung ist jedoch die Kenntnis einer Reihe von Faktoren notwendig, die heute offenbar nur für wenige $\mathrm{Ab}$ wässer bekannt sind. Die allgemeine Einführung der Formel wird dann möglich sein, wenn vermehrte Angaben über die C- und N-Verhältnisse im Abwasser vorliegen.

7. Die Berechnung der Beckengrössen von Belebungsanlagen und des Energiebedarfs für den Lufteintrag ergab, dass bei einem Reinigungseffekt von $80 \%$, gemessen am $\mathrm{BSB}_{5}$, der Energiebedarf für alle in der Praxis gebräuchlichen Beckengrössen nahezu konstant ist. Bei einem Reinigungseffekt von $90 \%$ hingegen nimmt der Energiebedarf bei grösseren Becken, bzw. bei längeren Belüftungszeiten, zu. Vom wirtschaftlichen Standpunkt aus betrachtet sind somit kleine Becken am günstigsten. Da jedoch mit abnehmender Beckengrösse, die zur Aufrechterhaltung des gewünschten Reinigungseffektes erforderliche Schlammkonzentration im Belüftungsbecken zunimmt, verhindert der praktisch erreichbare Schlammgehalt im Becken von selbst die Wahl zu kleiner Beliuftungsbecken, namentlich bei hohen Reinigungseffekten.

8. Wir sind der Auffassung, dass die von einigen kantonalen Gewässerschutzämtern empfohlenen Beckengrössen von einer Stunde Belüftungszeit bei Teilreinigung und von zwei Stunden bei Vollreinigung als Richtschnur angenommen werden dürfen, wobei diese Belüftungszeiten für die Belastung während der Tagesstunden angenommen werden. Rechnet man mit Mittelwerten des Tagesanfalls, so ergibt sich für das Abwasser der Stadt Zürich bei einer Stunde Belüftungszeit und 8oprozentigem Reinigungseffekt eine erforderliche Schlammkonzentration im Beliuftungsbecken von rund $2000 \mathrm{~g}$ Trockensubstan $z$ pro $\mathrm{m}^{3}$ Beckeninhalt. Bei zwei Stunden Belüftungszeit und einem 9oprozentigen Reinigungseffekt ist hingegen eine Schlammkonzentration von $4400 \mathrm{~g} / \mathrm{m}^{3}$ erforderlich. Beide Schlammkonzentrationen können in der Regel in der Praxis gut erreicht werden. Auf Grund der Versuche würde bei einer Schlammkonzentration von $3000 \mathrm{~g} / \mathrm{m}^{3}$ bei 
$80 \%$ Reinigungseffekt eine Belüftungszeit von knapp 40 min ausreichen, während ein 9oprozentiger Effekt eine Behandlung mit drei Stunden Belüftungszeit erfordert.

9. Ein Zusammenhang zwischen der Betriebsweise der Belebtschlammanlage und der Überschußschlammproduktion konnte nicht eindeutig nachgewiesen werden. Man wird deshalb vorerst noch, wenigstens für Anlagen mit Belüftungszeiten von $1 / 2$ bis $21 / 2$ Stunden, und für relativ dünnes Rohwasser, mit gleich grossen Überschußschlammengen rechnen müssen. Als Überschußschlammproduktion ergab sich $0,62 \mathrm{~kg}$ Trockensubstanz pro $\mathrm{kg}$ $\mathrm{BSB}_{5}$-Zufluss im Winter, bzw. $0,68 \mathrm{~kg}$ TS $/ \mathrm{kg} \mathrm{BSB}$ B $_{5}$ Zufluss im Sommer ohne die Schwebestoffe im Anlagenabfluss. Dabei betrugen die Vertrauensgrenzen für $\mathrm{P}=0, \mathrm{I}$ rd. \pm 0, IO $[\mathrm{r}]$.

Io. Für die Wahl der Höhenlage und der Länge der Überfallkanten von Belüftungsbecken ergeben sich folgende Richtlinien:

Bei Druckluftbelüftung ist die Überfallkante möglichst lang auszubilden, um die täglichen Schwankungen des Wasserspiegels im Belüftungsbecken zu begrenzen. Der Luftdurchsatz soll für den maximalen Wasserspiegel im Becken bemessen werden. Namentlich Systeme mit hochliegenden Beliuftungsrosten sind auf Wasserspiegelschwankungen empfindlich und müssen den Tagesschwankungen im Abwasseranfall besonders sorgfältig angepasst werden.

Bei Walzenbelüftung ist die feste Überfallkante der Überfallkrone beim Beckenauslauf mindestens $5 \mathrm{~cm}$ unterhalb Unterkante der Belüftungswalze anzuordnen. Die Überfallkante ist in Felder aufzuteilen, deren Überfallhöhe einzeln durch «Dammbalken» reguliert werden kann. Zwei dieser Felder sind so lang auszubilden, dass jedes für sich eine selbständige Steuerung der Eintauchtiefe der Walze bewirkt, so dass der Sauerstoffeintrag stets zur Deckung des Sauerstoffbedarfs bei einem goprozentigen Reinigungseffekt genügt. Hierfür dürfte eine Úberfallkantenlänge von weniger als $10 \mathrm{~cm}$ pro I000 angeschlossene Einwohner in den meisten Fällen ausreichen.

II. Zum Schluss möchte ich PD Dr.K.WUHRMANN (Versuchsleiter) und Dr.E. MärKI und seinen Mitarbeitern danken für die grosse Arbeit, die bei der Durchführung und Auswertung der Grossversuche geleistet wurde, sowie dem Werkstättechef E. FRIEDERICH und seinen Mitarbeitern, die während Jahren bei jeder Witterung, Sommer und Winter, die Versuchsanlagen zur vollen Zufriedenheit betrieben haben. "Last not least» gebuihrt unser Dank auch Prof.Dr.O.JAAG, welcher uns in der Gestaltung und Durchführung der Versuche die notwendige Freiheit lässt und die Finanzierung der Versuchsobjekte ermöglicht. 


\section{LITER A TUR VERZEICHNIS}

[I] K. WUHRMANN, Hauptwirkungen und Wecbselwirkungen einiger Betriebsparameter im Belebtschlammsystem; Ergebnisse mebrjähriger Grosspersucbe, Schweiz. Z. Hydrol. 26, Fasc. 2, S. 218 (1964).

[2] K.H. HuNKEN, Untersucbungen über den Reinigungsverlauf und den Sauerstoffperbraucb bei der Abwasserreinigung durch das Belebtscblammverfabren, Stuttg. Ber. zur Siedlungswasserwirtschaft H. 4, (Verlag R. Oldenbourg, München 1960).

[3] Klaus R. IMHOFF, Zur Berecbnung der Belebungsbecken GWF 104, H. 52, I494-I 498 (1963).

[4] A. HörLer, Ergebnisse pon Versuchen der EAWAG mit dem Kombibecken, Schweiz. Z. Hydrol. 26, Fasc. 2, S. 428 (1964).

[5] K. WUHRMANN, Grundlagen für die Dimensionierung der Belüftung bei Belebtscblammanlagen, Schweiz. Z. Hydrol. 26, Fasc. 2 ,S. 3 10 (1964).

[6] W. VON DER EMDE, Die Tecbnik der Belüftung in Belebtschlammanlagen, Schweiz. Z. Hydrol. 26, Fase. 2, S. 388 (1964).

[7] A.L. DownING, und A.P. Hopwood, Some Observations on the Kinetics of Nitrifying Activated-Sludge Plants, Schweiz. Z. Hydrol. 26, Fasc. 2, S. 27 I (1964).

Separatdruck aus der Schweiz. Zeitschrift für Hydrologie 26, Fasc. 2 (I964) Herausgegeben mit Unterstützung der Stiftung der Wirtschaft zur Förderung des Gewässerschutzes in der Schweiz 